\title{
ELT imaging and photometry in crowded fields
}

\author{
Peter Linde ${ }^{1,2}$ and Arne Ardeberg ${ }^{1}$ \\ ${ }^{1}$ Lund Observatory, Lund University, email: arne@astro.lu.se \\ ${ }^{2}$ Malmö University, email: peter@astro.lu.se
}

\begin{abstract}
The capability of extremely large telescopes (ELTs) to resolve stellar populations in distant galaxies has been investigated through simulation. With real stellar clusters as templates, images were created using a realistic point spread function model. Through variation of parameters such as aperture, Strehl ratio and exposure, as well as use of various stellar populations and densities, we study ELT capability to probe galaxy formation and evolution history. We conclude that a $50 \mathrm{~m}$ ELT should allow study of these processes in the Virgo galaxy cluster.
\end{abstract}

Keywords. Telescopes; Techniques: image processing, photometry; Galaxies: star clusters

\section{Introduction}

Revolutionary advances in astronomy can be expected from the introduction of the extremely large telescopes (ELTs). One of the science cases selected for special study is that of resolved stellar populations. A particularly important point is whether an ELT will allow disentangling of the details of galaxy evolution through observations of individual stars. A primary target for such observations is the Virgo galaxy cluster.

We have chosen to simulate clusters as seen with ELTs at different distances, investigating the influence of technical and observational factors such as telescope aperture, Strehl ratio, exposure time and object crowding. Here, we study imaging and photometric accuracy, while the astrophysical implications, particularly for different populations, ages and metallicities, are discussed in Ardeberg \& Linde (2006b).

\section{Simulations}

\subsection{Template clusters}

For our study, we chose two real clusters as templates, the open cluster NGC 6192 and the globular cluster M15, with ages of 0.7 Gyr and $11 \mathrm{Gyr}$. They were placed in a realistic background stellar population. The open cluster was created in two sizes with different stellar density, in the following referred to as as-is and extended versions. We used Strömgren photometry, appropriate for obtaining abundance data.

\subsection{PSF model and simulation parameters}

We assume that the technical challenges of adaptive optics (AO) system have been solved. The point spread function (PSF) in this case is basically determined by the telescope aperture and the Strehl ratio of the AO system. This sets the limits to achievable image quality, in terms of resolution and contrast. We adopted a PSF model formulated by Jolissaint \& Veran (2002) and further discussed by Andersen et al. (2003). The PSF shape is characterised by a diffraction limited central "hole", and a halo effect from the 

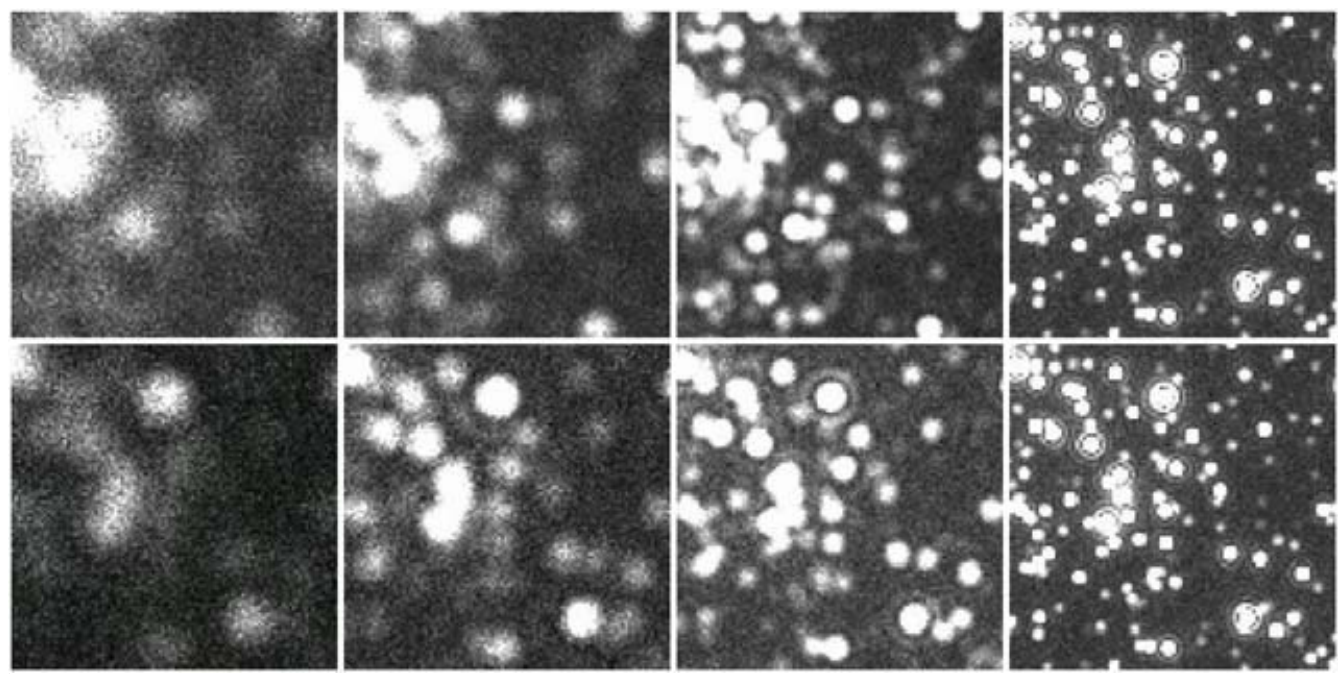

Figure 1. Region near the centre of the open cluster, as seen at $16 \mathrm{Mpc}$ with an AO ELT. The upper row shows the "as-is" version with apertures of 20,30, 50 and 100m, respectively. The lower row gives the corresponding sequence for the extended version.
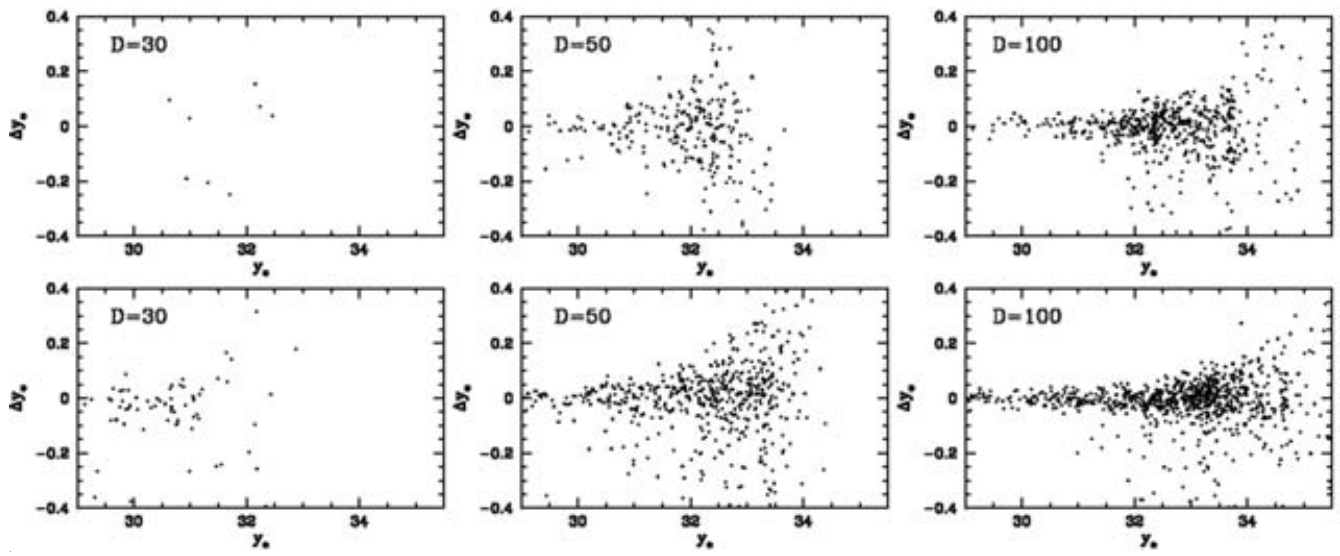

Figure 2. Photometric errors for the 30,50 and $100 \mathrm{~m}$ cases of the open cluster simulation. $y_{\mathrm{o}}$ is apparent magnitude and $\Delta y_{\mathrm{o}}$ is the difference between real and measured magnitude. Upper row: results for the "as-is" cluster version, lower row: extended version.

uncorrected outer parts of the seeing limited PSF. Various effects and limitations are discussed in Linde \& Ardeberg (2006).

The adopted field of view was 1 arcsec using a sampling of 0.5 milliarcsec per pixel. This allows single-conjugate AO and sufficient sampling for apertures up to $100 \mathrm{~m}$. The exposure time was in all cases 8 hours per filter band. A full description of the simulation parameters is given in Linde \& Ardeberg (2006).

\subsection{Images created and photometric analysis}

Using software developed in-house, several sets of $b, v$ and $y$ images were created to investigate the various effects mentioned above. The PSF shape peculiarities explain the halo effect seen around each individual star. This effect may appear damaging to the photometric measurements. However, as the cluster is seen at progressively larger 
distances, the individual halos merge into a common halo, as the cluster itself gets more point-like. Beyond $5 \mathrm{Mpc}$, the whole cluster is typically inside the diffraction-limited zone while the outside halo gradually vanishes into the background noise. Illustrations are given in Linde \& Ardeberg (2006) and Ardeberg \& Linde (2006a).

The simulated images were subsequently treated as observed images and submitted to photometric analysis. The DAOPHOT software package (Stetson, 1986) was applied in a fully automatic mode. The PSF was derived from images with somewhat lower image crowding than the actual cluster images. Some effort was made to optimise the DAOPHOT settings but no measurements were discarded.

\section{Telescope aperture}

The importance of telescope aperture was investigated with the open cluster at $16 \mathrm{Mpc}$. Simulated images in two sizes (densities) were created for apertures 10-100m, using Strehl ratio 0.6. While full fields for the clusters are shown in Linde \& Ardeberg (2006), we show in Fig. 1 the near-central parts in some detail, to facilitate evaluation of image quality. The corresponding photometric accuracy is shown in Fig. 2, where errors in individual measurements are shown versus apparent magnitude. We conclude that a $30 \mathrm{~m}$ aperture is only marginally useful, and then only in the less crowded, extended, case. The 50m case is much better as is, of course, the $100 \mathrm{~m}$ case. Corresponding colour-magnitude diagrams for the $50 \mathrm{~m}$ case are given in Ardeberg \& Linde (2006c).

\section{Strehl ratio}

As seen from theory (Andersen et al., 2003), the Strehl ratio is essentially determined by the seeing and the actuator spacing of the flexible AO mirror. In our simulations, we have varied the actuator spacing to obtain different Strehl ratios. Fig. 3 shows the effect of different Strehl ratios on images of the open cluster at $16 \mathrm{Mpc}$. In all cases, the aperture was $50 \mathrm{~m}$. The same sub-field is shown as in Fig. 1, with the as-is case in the upper row and the extended cluster in the lower row. Fig. 4 shows corresponding error diagrams. For comparison, we also show results for the $4 \mathrm{Mpc}$ simulation. Since even low Strehl ratios allow for a high resolution core of the PSF, the image analysis problem mainly becomes one of signal-to-noise-ratio. With decreasing Strehl ratios more photons are lost from the central PSF peak and image contrast decreases. This could largely be compensated through increased exposure. However, the crowding effect is the more serious one. More discussion is found in Ardeberg \& Linde (2006b).

\section{Globular cluster}

The simulated globular cluster mimics M15 in stellar density and age. Hence, its member stars are intrinsically considerably fainter than those of NGC 6192. Also, stellar crowding is even more serious. Fig. 5 shows the cluster at 5,8 and $16 \mathrm{Mpc}$. A sub-field is seen in Fig. 6. Also in this case, most of the background light, from uncorrected PSF wings, is in a merged halo outside the cluster itself. The photometric precision, seen in Fig. 7, clearly suffers from the combination of crowding and lower flux.

\section{Conclusions}

The simulations show that in spite of unusual PSF properties, adequate data for studies of galaxy evolution can be obtained using well-known procedures. The residual PSF halo 

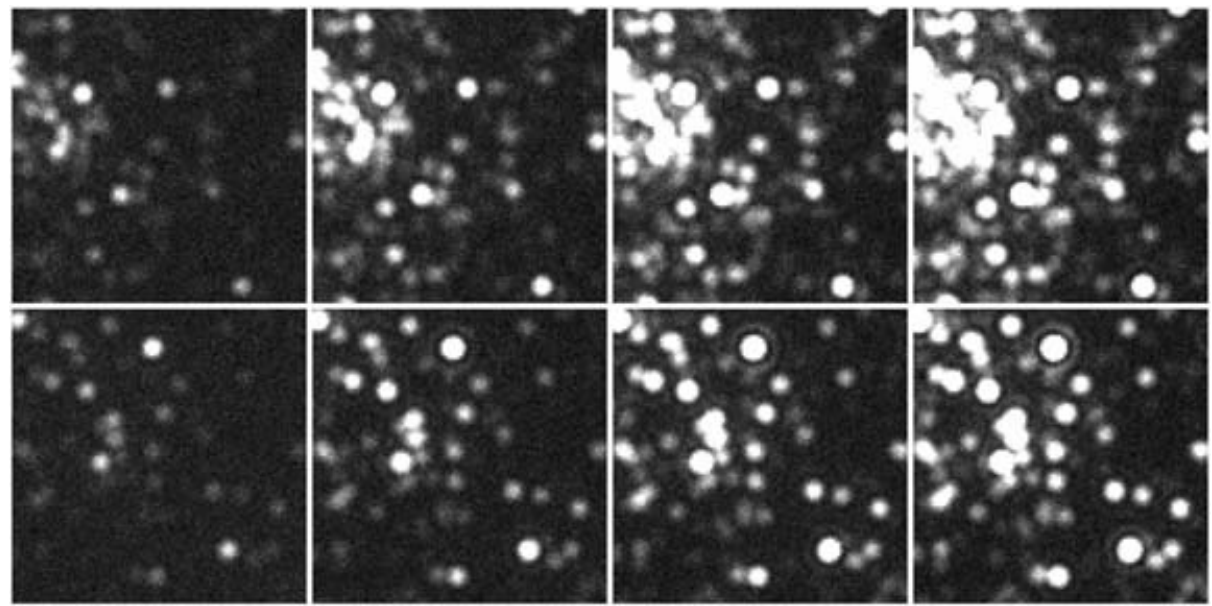

Figure 3. Region near the centre of the open cluster, at $16 \mathrm{Mpc}$ with a $50 \mathrm{~m}$ AO ELT. The upper row shows images of the "as-is" version with Strehl ratios of $0.2,0.4,0.6$ and 0.8, respectively. The lower row gives the corresponding sequence for the extended version.
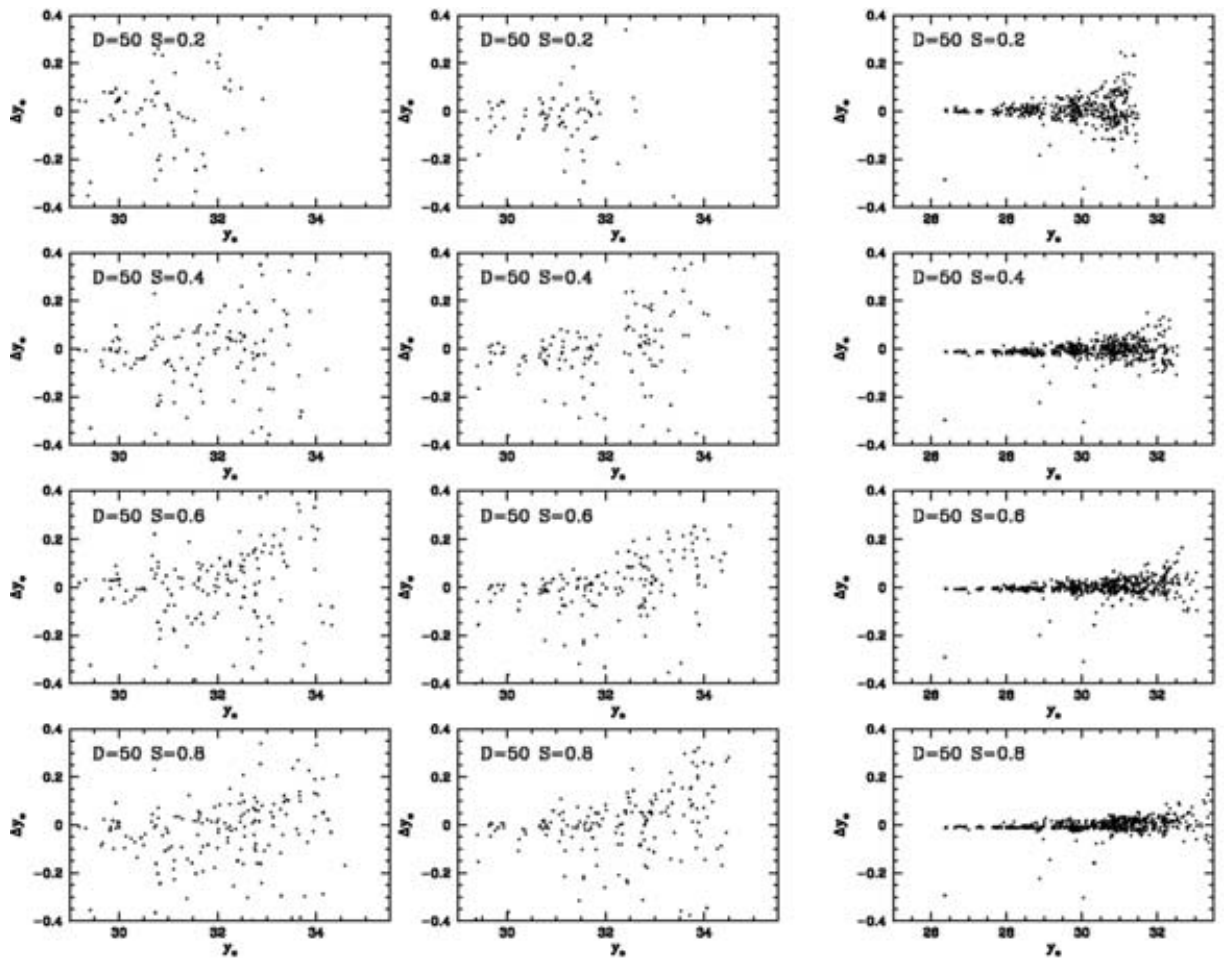

Figure 4. Error diagrams as in Fig. 2, but derived using Strehl ratios 0.2, 0.4, 0.6 and 0.8 for a $50 \mathrm{~m}$ AO ELT. The left and middle columns show the "as-is" and extended cluster data for $16 \mathrm{Mpc}$. The right column shows data for the extended cluster at $4 \mathrm{Mpc}$.

problem can largely be avoided when observed objects are sufficiently small to fit into the diffraction limited region. We conclude that crowding is more serious than photon starvation. This means that, not surprising, resolution is paramount for resolving stellar populations in the Virgo galaxy. A lower Strehl ratio can, to some limited extent, be 

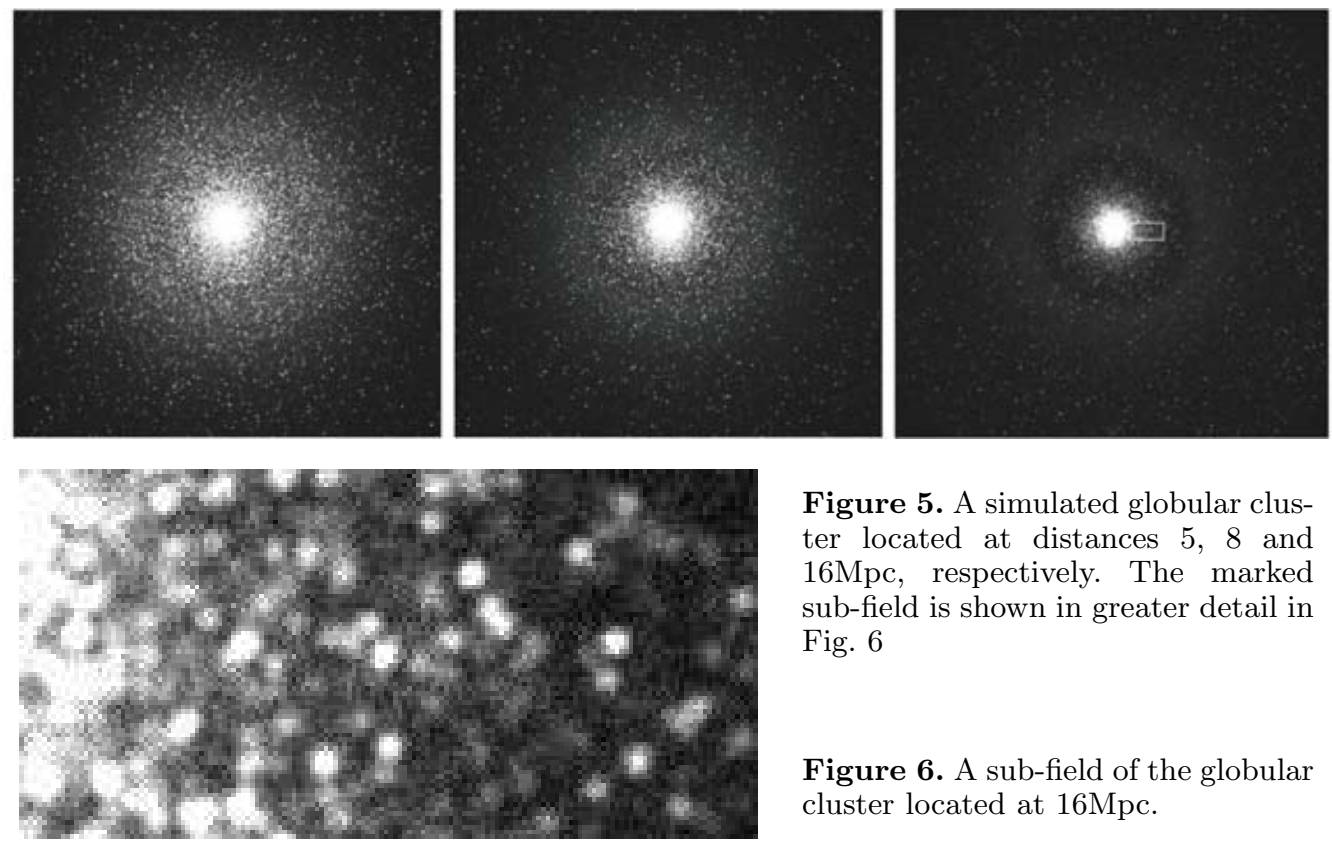

Figure 5. A simulated globular cluster located at distances 5,8 and $16 \mathrm{Mpc}$, respectively. The marked sub-field is shown in greater detail in Fig. 6

Figure 6. A sub-field of the globular cluster located at $16 \mathrm{Mpc}$.
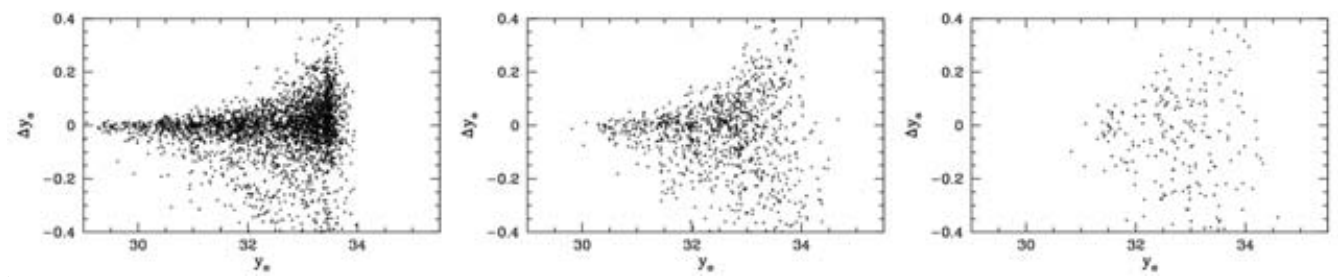

Figure 7. Error diagrams for globular cluster data. Distances are 5, 8 and 16Mpc.

compensated for through increased exposure. With a 50m aperture, resolution is sufficient in most cases. For younger stellar populations, flux levels are satisfactory while older stellar populations will need exposures in excess of 8 hours.

Our further simulation work will include effects of temporal and spatial variability (see Olsen 2006). This will complicate the photometry. On the other hand, we see room for further improvement in the extraction of photometric information.

\section{References}

Andersen, T., Ardeberg, A. \& Owner-Petersen, M. 2003, Euro50: A 50m Adaptive Optics Telescope, Lund Observatory, Sweden.

Ardeberg, A. \& Linde, P. 2006a, Proc. Workshop. Instr. for ELTs, Ringberg 2005, in press. Ardeberg, A. \& Linde, P. 2006b, Proc. Workshop. Instr. for ELTs, Ringberg 2005, in press. Ardeberg, A. \& Linde, P. 2006c, this volume.

Jolissaint, L. \& Veran, J-P. 2002, E. Vernet, R. Ragazzoni, S. Esposito \& N. Hubin (eds.), ESO Conference and Workshop Proceedings 58, 201.

Linde, P. \& Ardeberg, A. 2006, Proc. Workshop. Instr. for ELTs, Ringberg 2005, in press.

Olsen, K. 2006, this volume.

Stetson, P.B. 1987, PASP 99, 191. 


\section{Discussion}

Piotтo: I am pretty sure that Daophot is not the most appropriate software for "accurate" PSF fitting photometry on images from AO. Other software is available and it is conceivable that we need specific software to be written. However, I have two questions: 1) How stable in space is the PSF? To do PSF photometry, you have to define your PSF, and if it changes too quickly, moving out from the cluster centre, you might have not enough stars to calculate the PSF. 2) More importantly, how stable is the PSF in time? In order to have quantitative information we need to transform our instrumental magnitude into some absolute quantity, and for this PSF stability in time is mandatory.

LINDE: We have not yet studied these variabilities in detail, although, undoubtedly, they are quite important. However, we believe that spatial PSF variation should be quite limited in the small fields $(\approx 1$ arcsec $)$ that we are using. Natural seeing temporal variability will affect the Strehl ratio, as will, of course, the wind effect. I agree that more specialised software should be developed and that it will improve the photometric extraction accuracy.

ARDEBERG: First, I agree that more dedicated software would be very helpful. Second, we should realise that our problem is rather complex with many parameters involved. These have to be considered for a substantial improvement. To be able to give an adequate comment regarding photometric precision, in time and over the field, with AO systems, we need end-to-end modelling. In the Euro50 project, such a model exists and it can be run in closed-loop version. Thus we can study optical path differences (OPDs) So far, our results are far from satisfactory. However, they do improve.

Gouliremis: 1) Did you find any difficulty in applying "classical" crowded field photometry packages to your simulated observations? 2) Did you consider that you might compromise lower Strehl ratio for longer exposures?

LINDE: In fact, we feel that so far classical techniques are adequate, while there is still room for improvement in the photometric analysis. It is correct that lower Strehl ratios partly can be compensated for by using longer exposure times.

OLSEN: There is no question that having AO corrections producing Strehl ratios of 0.6 in the V-band would be a great boon for stellar populations. But my impression is that such systems are technically out of reach.

ARDEBERG: Expectations regarding Strehl ratios are often optimistic, I agree. We feel that a reasonable assumption is that an ELT, such as the Euro50, will initially reach an AO wavelength limit of 1-2 $\mu \mathrm{m}$. Strehl ratios should reach the 0.6 level. With some further development over some further years, such Strehl ratios should be achievable also in the visual wavelength region.

DAVIDGE: Just a comment about the issue of calibration raised in an earlier question. A major source of uncertainty will be the energy in the PSF wings at annular scales of 0.5-1.5 arcsec. The fields of view of the images used on ELTs will have to be a few arcsec to monitor this light level. 